\title{
Carcinogenesis with mitochondrial respiratory degeneration was testified using a specific herbal medicine
}

\section{Tsuneo Kobayashi*}

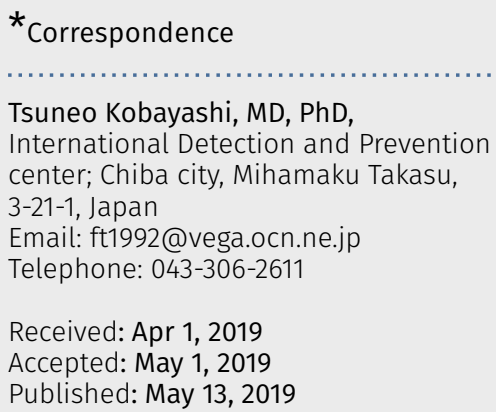

\begin{abstract}
Background: Oncologists continue to dispute whether the main factors underlying carcinogenesis are related to genetic abnormalities or rather protracted mitochondrial respiratory dysfunction, both are core reasons for the occurrence of carcinogenesis. Thus, the aim of this article is to demonstrate that mitochondrial respiratory degeneration might be the true causal factor underlying carcinogenesis by presenting evidence obtained from experiments with a specific herbal medicine to inhibit oxygen respiration in cancer cells.
\end{abstract}

Methods: The tested herbal medicine (Sun Advance) was used to selectively impact mitochondrial respiration of cancer cells,

which is a hallmark of cancer, the results would thereby obtain evidence that carcinogenesis originates from mitochondrial respiratory degeneration.

Results: In cellular and animal experiments, the Sun Advance had good effects against most hallmarks of cancer.

Conclusion: Carcinogenesis is a form of disease initiation due to mitochondrial respiratory degeneration testified using a specific herbal medicine. This demonstrate that mitochondrial respiratory degeneration might be re-differentiating factor with the use of herbal medicine. Therefore, primary cancer prevention programs should advocate for life-style changes and dietary energy restriction.

Keywords: Genetic abnormalities, Protracted mitochondrial respiratory degeneration, Genetic instability, Herbal medicine, Cancer cell respiration, Defective immune-surveillance.

\section{Introduction}

About 60 years ago, Otto Warburg initially proposed that aerobic glucose fermentation was an epiphenomenon representing one of the most fundamental problems in cancer cell biochemistry [1] that is impaired or damaged respiratory dysfunction. But many oncologists believe that carcinogenesis originates from a number of genetic abnormalities, but Weinhouse and Alan [2-4] have criticized Warburg's insisting. Although, Peter Pedersen and Seyfried \& Shelton have shown that protracted mitochondrial respiratory dysfunction is the main mechanism underlying carcinogenesis. However, much of the confusion surrounding the origin of cancer arises from lack of a unifying theory that can integrate the diverse observation on the nature of the disease. Seyfried \& Shelton showed that the majority of cancer gene defects could arise as downstream epiphenomenon of tumor progression, rather than as cancer causes [5,6].

Damage to the mitochondria which causes a loss of cytochrome $\mathrm{C}$ from the associated mitochondria, is understood to subsequently prevent cell from the undergoing apoptosis. Furthermore, hybridization experiments have confirmed that the mitochondria of normal cell have the ability to overwhelm those of cancer cells. 
Koura $\mathrm{H}$. et al. experimented with the hybridization of enucleated normal cells with cancer cells and found that the hybridized cell (cybrids) reverted to normal cells because the normal mitochondria were sufficiently powerful to overtake the nuclear DNA of cancer cells. Researchers who insist that carcinogenesis is induced by the accumulation of genetic abnormalities have neglected the results of these hybrids (cybrids) experiments as described bellow (Figure 1) [7-11].

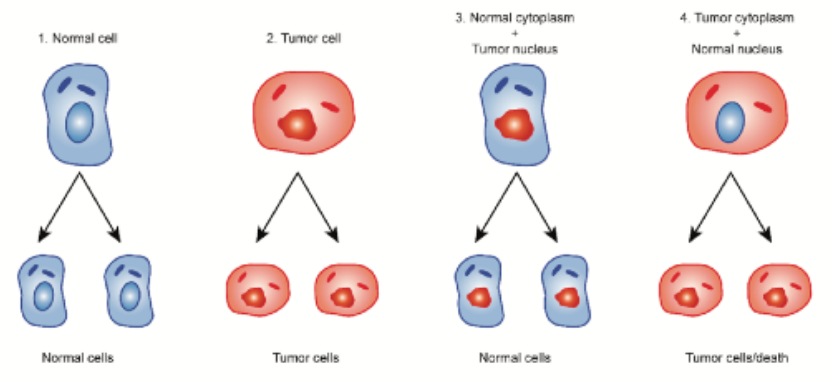

Figure 1: Cybrids experiments cited from Koura et al.

Seyfried et al. reported that normal mitochondrial function is maintained in a differentiated state, thereby suppressing carcinogenesis, whereas dysfunctional mitochondria can enhance dedifferentiation, thereby facilitating carcinogenesis (Figure 2).

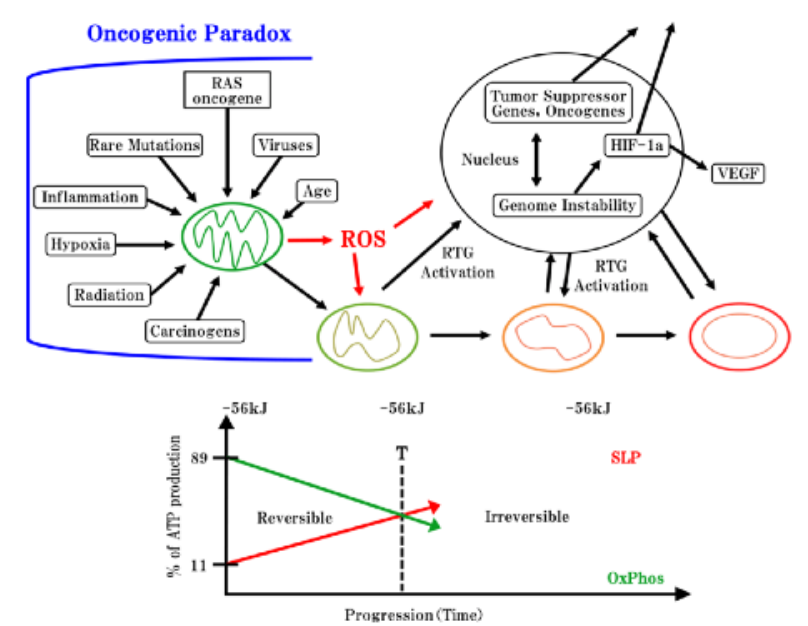

Figure 2: Oncogenic paradox, mitochondrial degeneration processing and down stream of genetic change cited from Thomas Seyfried et al.

However, mitochondria are involved in not only metabolic diseases, but also development diseases, as these organelles play a key role in cell differentiation [12-17]. Madhavika et al. found that mature mitochondria are degraded or fragmented by oncogenic MAPK signaling, and that from fragmented mitochondria can form matured mitochondria by inhibition of oncogenic MAPK signaling [18].

In regard to the ghost-like respiratory dysfunction of mitochondria, a specific herbal medicine, Sun Advance (hereafter referred to as SA: IMHC, Chiba city, Japan) which is a mixture of 18 herbs has been investigated the molecular level for 10 years, but no molecular basis has been apparent, thus, the herbal medicine was manipulated as a single medicine for further research because this herbal medicine promotes a simple specific inhibiting function in cancerous oxidative respiration by selectively inhibiting oxygen consumption of Ehrlich ascites cancer cells. Hence, this herbal medicine could be used to demonstrate that mitochondrial respiratory degeneration is in reverse to the true mechanism underlying carcinogenesis. Many researchers have investigated carcinogenesis based on carcinogenic factors and subsequent molecular changes and dedifferentiation. However, mitochondrial function is closely related to molecular and morphological differentiation. Therefore, one of the aims of this article is to demonstrate that mitochondrial respiratory degeneration might be a differentiating factor with the use of SA which selectively inhibits the oxygen respiration of cancer cells according to Koch's rule. In addition, the effects of SA on the hallmarks of cancer are discussed in detail [19-22].

However, mitochondria are not only energy centers, but also centers of respiration and differentiation. Mitochondria evolved more than 1.8 billion years ago, thereby facilitating the evolution of anaerobic cells into aerobic higher energy eukaryotes that produce 38 ATP molecules of adenosine triphosphate (ATP) to produce the second messenger molecule cyclic adenosine monophosphate (AMP).

About $90 \%$ of mitochondrial respiratory DNA is inserted into nuclear DNA, and the other 10\% is inserted in mitochondria by an unknown mechanism. Mitochondria are fundamentally bacterial in origin; and molecular fusion and fission occur easily, using a completely different process from eukaryote DNA during mitosis. Fertilization in higher animals between an egg and sperm may represent the process when original mitochondria entered into large anaerobic bacterial cells about 1.8 billion year ago. On the basis of this hypothesis, the 
hybridization experiments(cybrids) of enucleated normal cells and cancer cells conducted by Koura' et al. should be considered as a type of fertilization $[9,15]$.

1. SA selectively inhibits oxygen respiration of Ehrlich ascites cancer cells via a combination of albumin, $\alpha$ and $\beta$-globulin and palmitate. Moreover, the factors that cause albumin to decrease and the $\alpha-, \beta-, \gamma-$ globulin fractions to increase with cancer progression will be investigated [23]. Additionally, relevant data related to albumin are presented in this paper.

a) The effects of a combination of albumin(10-4M)(A) + palmitate $\left(10^{-5} \mathrm{M}\right)(\mathrm{P})+\mathrm{SA}: \mathrm{S}(35 \mu \mathrm{g})$ are described in Figure 3.

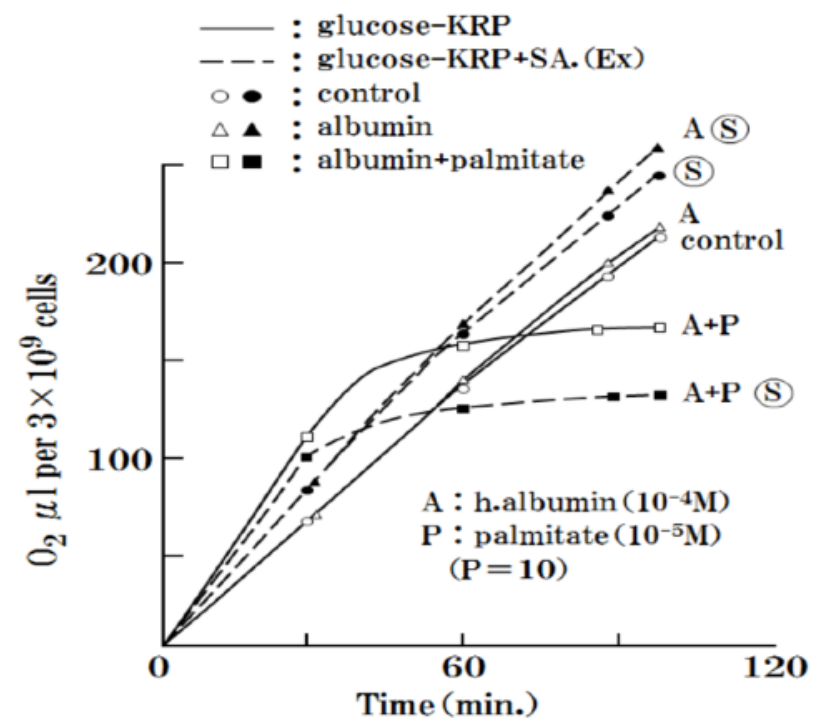

Figure 3: Oxygen respiration will suppressed by only the combination of $A+P+(S)$.

Oxygen consumption experiments were performed using co-occurring human-albumin $\left(10^{-4} \mathrm{M}\right)(\mathrm{A})$ and palmitate $\left(10^{-5} \mathrm{M}\right)(\mathrm{P})$ and $\mathrm{SA}(35 \mu \mathrm{g})(\mathrm{A}+\mathrm{P}+\mathrm{S})$ ), The results showed that the oxygen consumption of Ehrlich ascites cells was selectively inhibited, although, inhibition did not occur with SA alone(S) or with the combination of $S A$ and albumin( $A+(S)$.

Moreover, normal cells were not affected at all, as discussed below. Thus, the observed inhibition effects were a selective reaction in Ehrlich ascites cells.

The effects SA on normal cells $(\mathrm{C} 3 \mathrm{H}$, mouse kidney cell) are detailed in Figure 4. The extracted juices of apple and cucumber were used in the experiment because both are recommended with a clinical prescription.

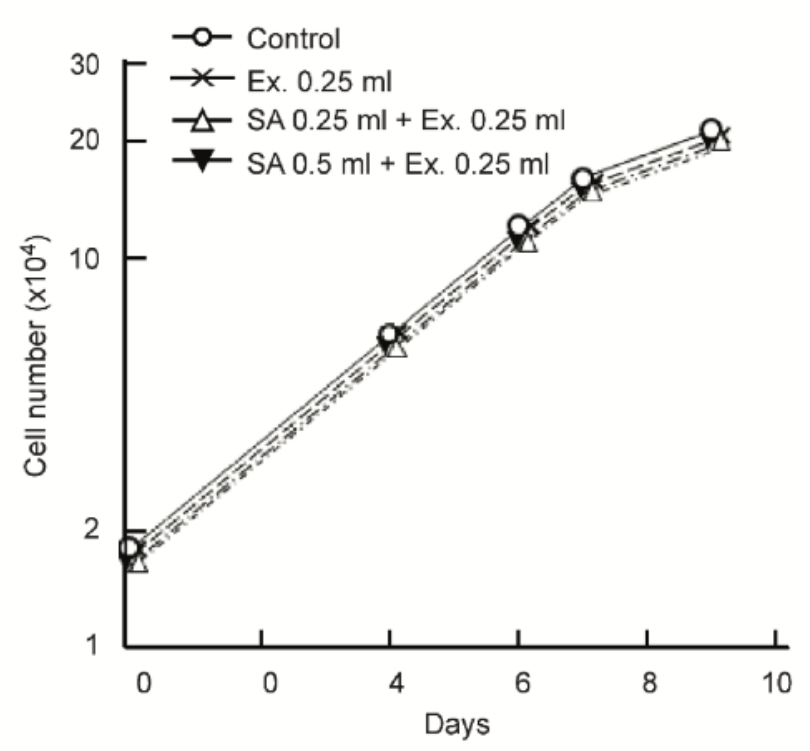

Figure 4: No effect of SA on normal cell.

2. On the basis of this research, SA likely has a direct effect on mitochondria by inhibiting respiration in cancer mitochondria, especially damaged mitochondria.

Here, the effectiveness of SA against most hallmarks of carcinogenesis is discussed.

1) $S A$ inhibits carcinogenesis by carcinogens.

2) SA inhibits metastasis.

3) SA inhibits and excludes toxohormone, which produces cachexia upon reaction in the serum.

4) SA activates lactate metabolism.

5) SA inhibits DNA-type of carcinogenesis via simian virus 40(SV40) infection.

6) SA inhibits carcinogenesis, but does not inhibit SV40 infection as determined in CV1 monkey kidney cells.

7) SA quantitatively inhibits growth of transformed cancer cells.

8) SA adjusts lipid metabolism

In the following text, each topic is demonstrated stepwise.

S-1) Chemical carcinogenesis in mice was induced using $0.4 \mathrm{mg}$ of 20 -methychorantherene (MC). As a strong chemical carcinogen $0.1 \mathrm{mg} M C \times 4$ times was administered via subcutaneous injection. The gray 
colored area indicates the times of oral SA $(4 \mathrm{mg} / \mathrm{ml})$ administration to the mice. Black circles indicate tumor-bearing mice. X indicates death before day 90 . Survival is the number of surviving animals at the conclusion of experiment/ the number of animals at the start of the experiment (Figure 5).

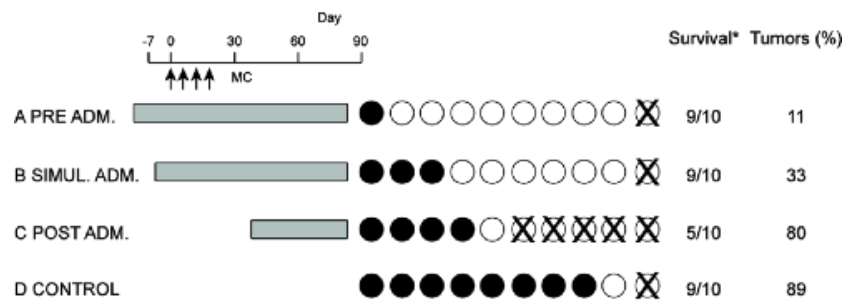

Figure 5: Carcinogenesis by carcinogen (MC) is suppressed by the addition of SA.

Longer durations of SA administration corresponded to longer life spans in the mice and reduced the proportion of mice that developed cancer to only $10 \%$. In the control (without SA administration), $80 \%$ of the mice developed cancer, and $10 \%$ died.

These data show SA had a strong inhibiting effect on carcinogenesis.

S-2) The inhibitory effects of SA on metastasis were investigated utilizing $\mathrm{C} 3 \mathrm{H}$ mice (10 mice per group). Briefly, after injection of MH134 cancer cells(5x106) into the tail veins.

For 14 days, the metastatic lymph-nodes were counted by autopsy investigation. Strangely and contrary to expectations, the administered dose of SA had effectively inhibited metastasis according to their doses. Furthermore, SA administration via oral intake in water was found to convey strong metastasis-inhibiting effects. (Figure 6).

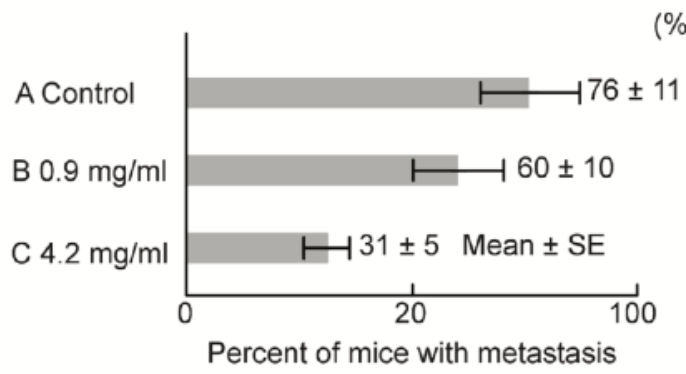

Figure 6: Metastasis is inhibited by the addition of SA according to their doses.

These results may be due to the strong respiratory inhibition of SA in cancer cells.
S-3) SA inhibits and excludes toxohormone, which results in cachexia upon reaction with serum (Figure 7).

SA was found to effectively inhibit the reduction of serum iron by toxohormone as described previously. "The time course of serum iron levels reduced by Toxohormone (T.H.)".

Marmots were used in this experiment because mice are too small for the required six blood collections (>3 ml) [21].

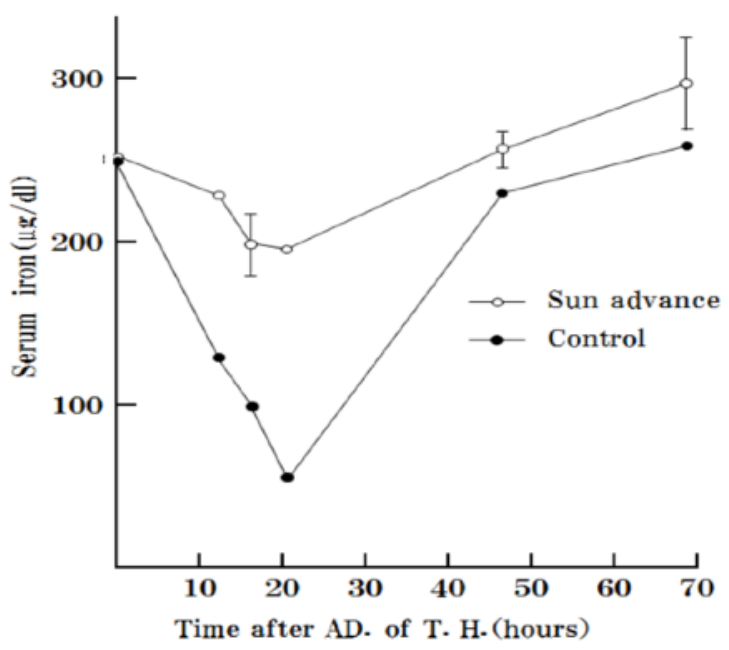

Figure 7: SA inhibits the effects of toxohormone and excludes toxohormone.

S-4) SA removes lactate from the tissues and then the serum is finally excreted in the urine (Figure 8).

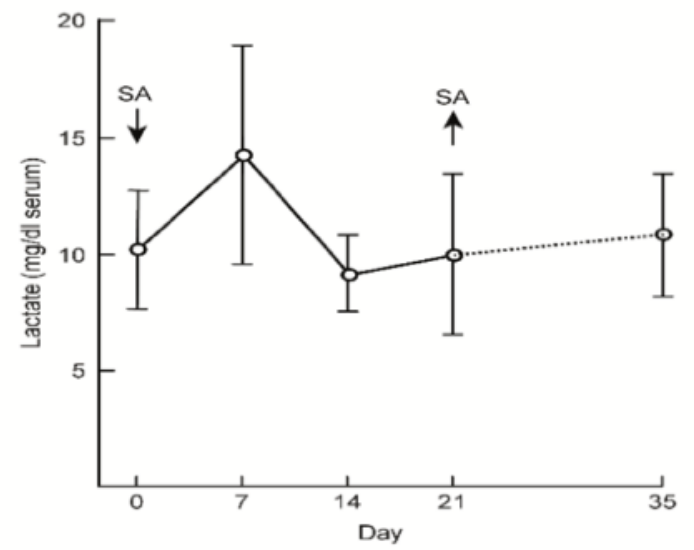

Figure 8: SA removes lactate from serum

These data represent the average data of five candidates who were administered SA (1.5g/day) daily, for 21 days. On the basis of these data, SA removed lactate from tissue, which led to an increase 
in serum lactate after 7 days, which was then excreted in the urine.

S-5) SV40 (DNA virus type) transformation experiments were performed using mouse kidney cells [22].

S-5-1) On the right side of the photograph in Figure 9, cell contact inhibition is clearly shown in the plate

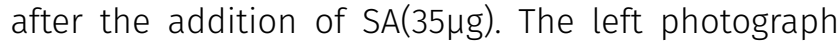
shows the aggregation of cells, which have been clearly transformed (Figure 9).
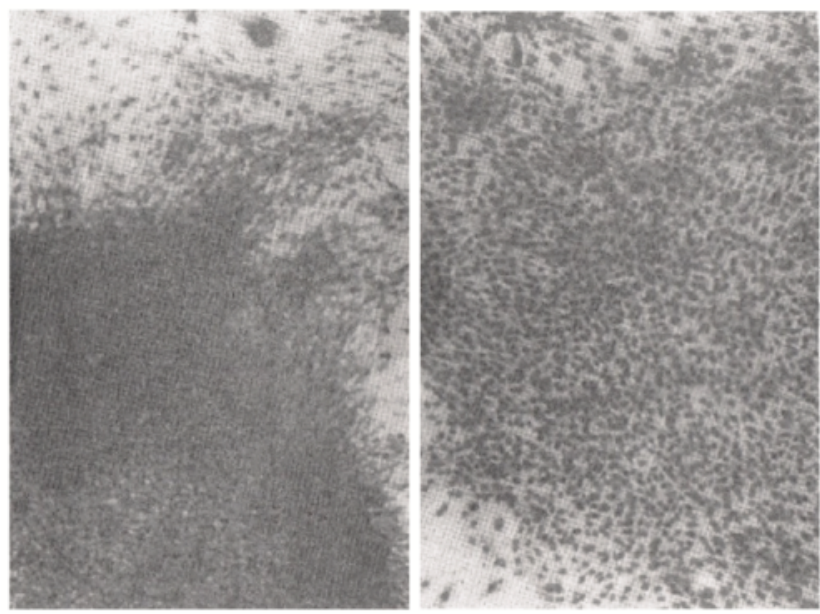

Figure 9: Contact inhibition is clearly shown using the addition of SA in carcinogenesis by SV40 virus infection.

S-5-2) In this Figure 10, $10^{3}$ mouse kidney cells were seeded on one plate and transfected with SV40 virus

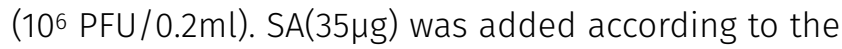
schedules A H (Figure 10).

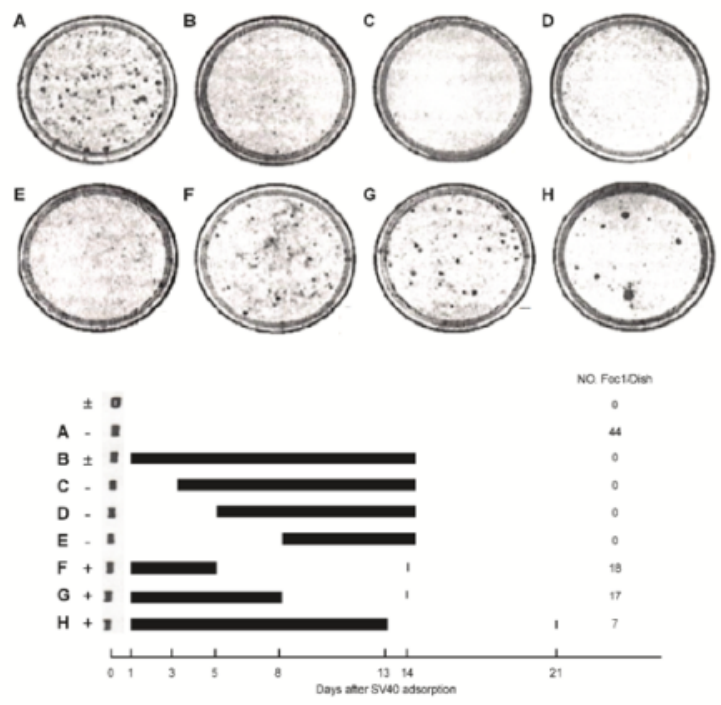

Figure 10: Transformation of SV40 virus infection as shown is reversibile by the addition of SA.
In the upper portion of Figure 10, transformed foci are observed in low Ca2 + medium, whereas, in the lower portion, the black bar indicates the addition of SA.

The data show that SA prevented SV40 transformation by reversing the transformation.

S-6) As shown by the graph in Figure 11, the growth of SV40 virus-transformed cancer cells was quantitatively inhibited by the addition of SA. Because SA inhibits damaged mitochondrial respiratory dysfunction, SA inhibits carcinogenesis before and after SV40 transformation (Figure 11).

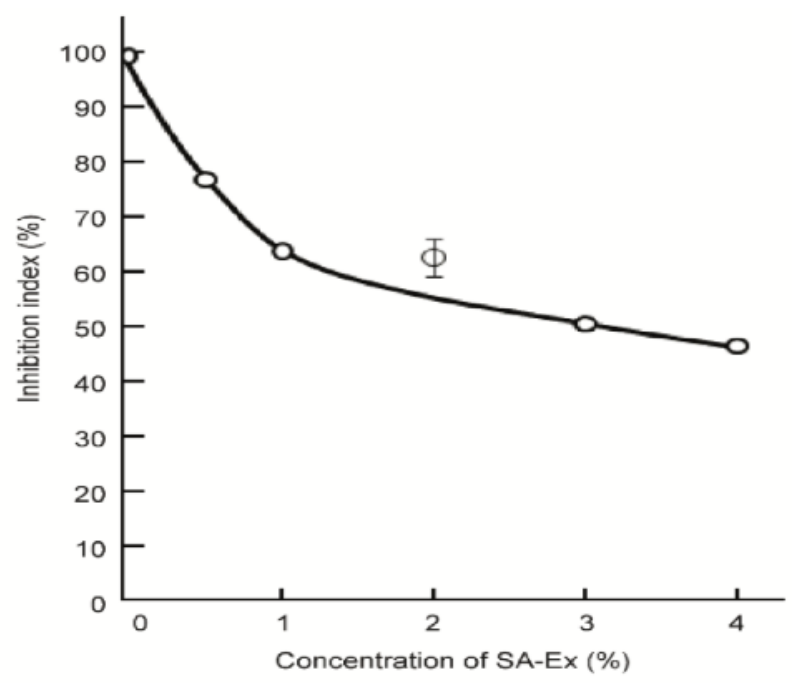

Fig 11: The grown up of SV40 virus transformed cells were inhibited quantitatively.

3. The Warburg effect may be misunderstood because it suggests that in normal cells, approximately $100 \%$ of the ATP is produced by oxidative phosphorylation, whereas in cancer cells, approximately $100 \%$ of the ATP is produced by glycolysis. Although Reitzer et al. reported that Hela cells require oxygen, the internal ATP levels were maintained by glutamine metabolism under aerobic conditions for up to $120 \mathrm{~min}$. This finding indicates that aerobic glycolysis and mitochondrial ATP production are necessary to maintain ATP concentrations, even in surviving cancer cells [24] (Figure 12).

Thus, the survival of cancer cells may be inhibited because the necessary internal ATP derived energy cannot be produced, which may explain how SA inhibits cell proliferation and transformation. 


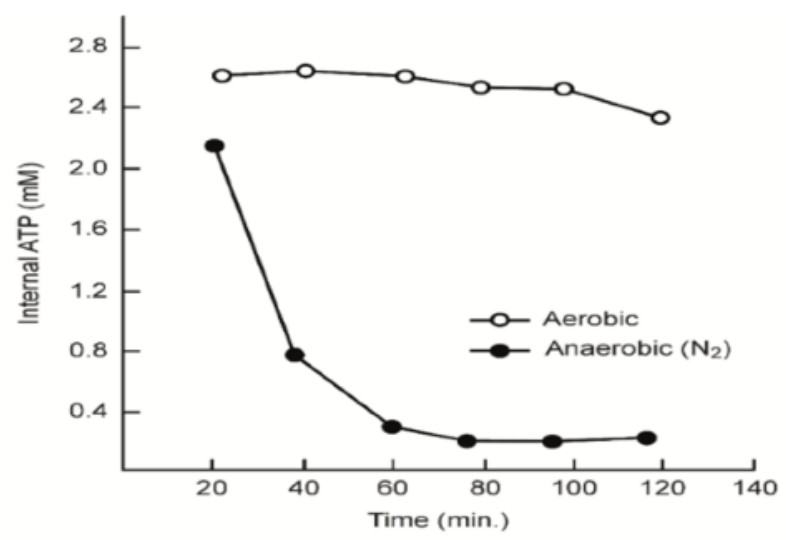

Figure 12: Strict anaerobic condition don't permit for cancer cell to live along with glucose cited from Reitzer et al.

4. However, in reality, $10 \%$ of their ATP in normal cells is produced via glycolysis and $90 \%$ via oxidative phosphorylation. Hence, these proportions may be correlated to the proportions of mitochondrial DNA actually in the mitochondria (10\%) versus that left in the nucleus $90 \%$. In contrast, in cancer cells, $60 \%$ of ATP is produced via glycolysis and $40 \%$ of ATP via oxidative phosphorylation, as indicated in the following Figure 13. Oxidative phosphorylation decreased to $40 \%$ along with mitochondrial respiratory function in cancer cells exhibiting dysfunctional changes into immortal cancerous cells [23]. It is reasonable that normal cells transform into immortal cells which have the more dominant form of energy production system via reversion from oxidative phosphorylation to the bacterial glycolysis, which is the original mode of bacterial energy production. It follows that changing of the energy production system, will result in a change in the cell division system from the eukaryotic type to the prokaryotic type (Figure 13).
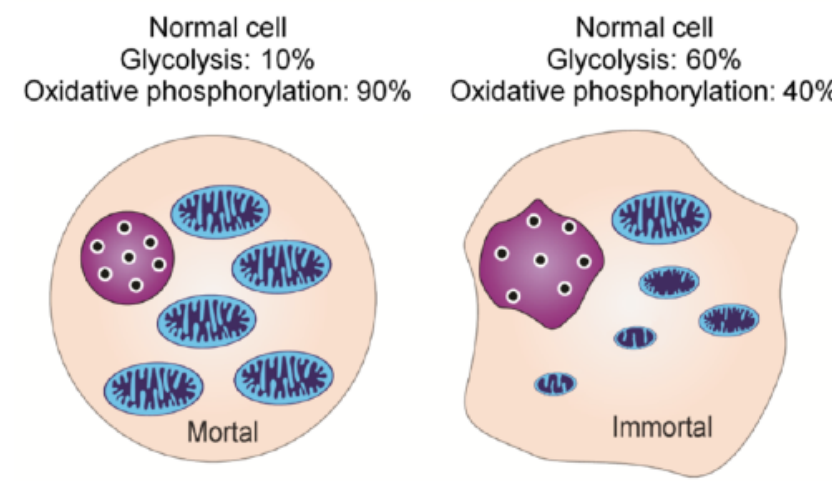

Figure 13: Figurative mitochondria in normal cell and cancer immortal cell.
On the basis of these findings, SA is thought to inhibit the remaining aerobic respiration of oxidative phosphorylation (Krebs cycle) in cancer cells. One must consider the biological puzzle as to why $90 \%$ of mitochondrial DNA is inserted into nuclear DNA and $10 \%$ of mitochondrial DNA remains in the mitochondria.

Therefore, decreasing the Krebs cycle to less than $40 \%$ would also decrease excessive inhibition of oxidative phosphorylation to less than $40 \%$. Thus, the survival of cancer cells may be inhibited because the necessary internal ATP energy is difficult to produce, which may explain why SA inhibits the proliferation and transformation of cancer cells.

Incidentally, Madhavika et al. reported that mitochondrial fragmentation is requisite to RASinduced transformation and metabolism of cancer cells. On the other hand, the targeting of maturing mitochondria with inhibitors against the oncogenic MARK pathway restored normal metabolism [18]. This reversible mitochondrial change may be correlated with the mitochondrial change mentioned above (Figure 14).

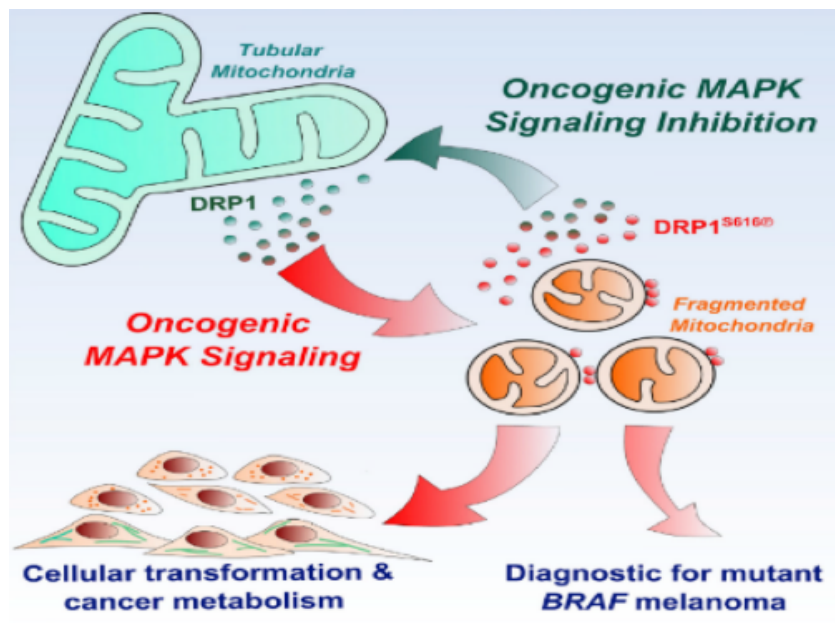

Figure 14: From mitochondria maturation to fragmentation of mitochondria vice versa is reversible cited from Madhavika et al.

5. Abe et al reported that the addition of Panax Ginseng saponins $(20 \mu \mathrm{g})$ led to the re-differentiation of Morris hepatoma cells into normal liver cells after 2 months [27] (Fig 15).

As shown by microscopic observation, hepatoma cells could re-differentiate into normal liver cells with the use of Panax ginseng saponins $(20 \mu \mathrm{g})$ after 2 months. 


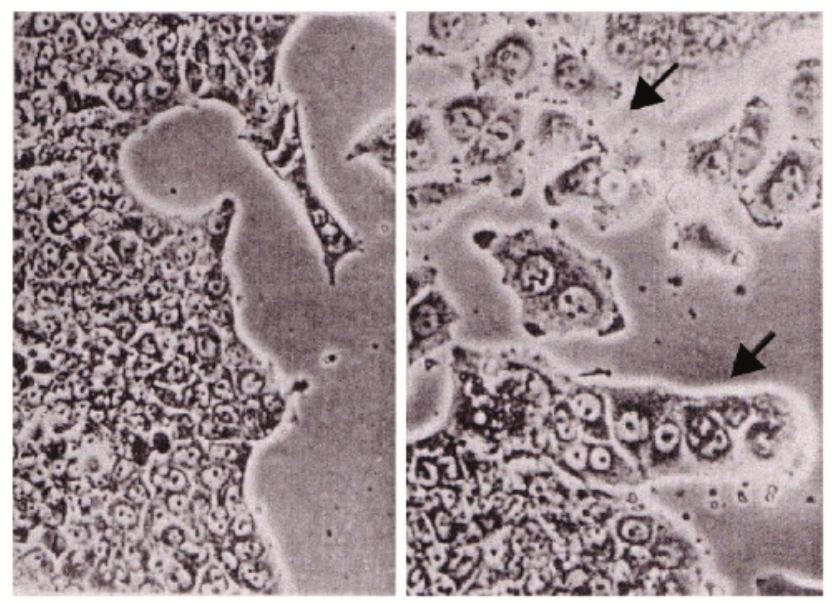

Figure 15: Morris hepatoma cells re-differentiated to normal liver cells by the use of Panax Ginseng cited from Abe et al.

Electron microscopic observation clearly showed that fragmented and shrunken mitochondria had clearly re-differentiated into normal mature mitochondria (Figure 16). Also, segregated and fragmented smaller mitochondria reverted to normal mature large mitochondria after the addition of Panax ginseng saponins $(20 \mu \mathrm{g})$ as shown in Figure 16.
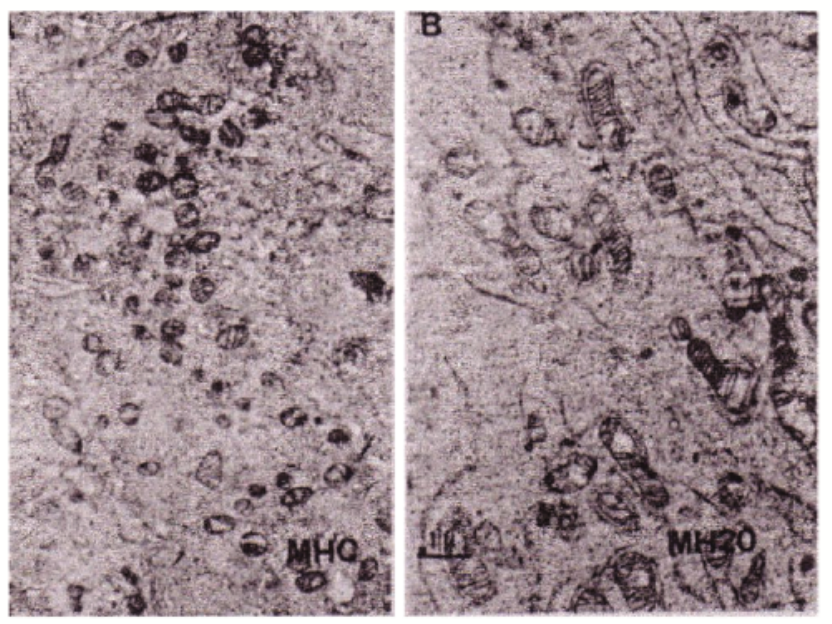

Figure 16: According to the differentiation of hepatoma, its mitochondria changed from fragmented type to matured mitochondria cited from Abe et al.

The result of the Panax ginseng experiment also suggested that there is no correlation between redifferentiation of Morris hepatoma cells and genetic abnormalities of cancer cells. In regard to the defective immune-surveillance of cancer patients, PD-1 and PD-L1, immune-checkpoint inhibition pathway was reported in a molecular biological study. Another biochemical cause of defective immune-surveillance will be demonstrated in the next chapter.

\section{Survey of cyclic AMP in cancer patients}

As confirmed in cancer and preclinical cancer patients (40) who were classified as TSIV and TSV according to the degree of cancer development, reduced $T$ cell immunity (Fig 17) and natural killer activity (Fig 18) resulted in lower serum levels of cyclic AMP [7,28]. The average cyclic AMP serum concentration was $16 \mathrm{pmol} / \mathrm{ml}$ with a lower T cell less than $1000 / \mu \mathrm{L}$. Conversely, the average cyclic AMP was $18.2 \mathrm{pmol} / \mathrm{ml}$ with a $\mathrm{T}$ cell number of $>1000 / \mu \mathrm{L}$. These phenomena are fundamental biological characteristics of defective immune-surveillance in cancer patients.

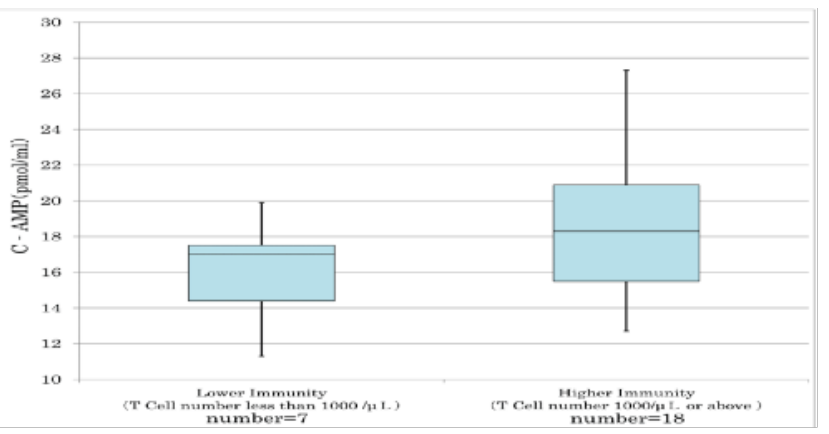

Figure 17: The correlation between T cell number and cyclic AMP in serum.

The average cyclic AMP content is $14.5 \mathrm{pmol} / \mathrm{ml}$ in natural killer cell less at less than 30\% activity, and $19 \mathrm{pmol} / \mathrm{ml}$ at activity of $30 \%$ or above.

This phenomenon also illustrates the fundamental biochemical background of defective immunesurveillance in cancer patients.

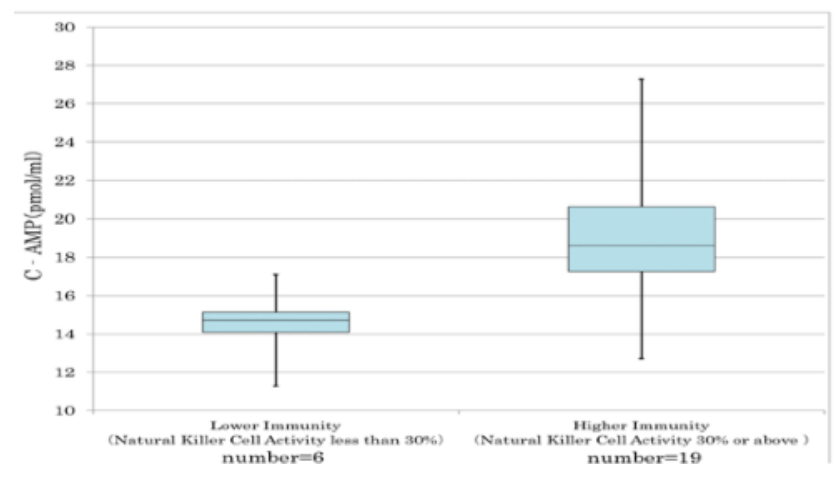

Figure 18: The correlation between NK activity and cyclic AMP in serum.

According to these data, there is a correlative tendency between lower immune activity and lower 
cyclic AMP serum concentrations following cancer development.

7. In this section, direct effect of cyclic AMP on cancer cells in tissue culture is reported.

A study conducted at the University of Colorado (Denver Co, USA) by Hsie et al. demonstrated that $1 \mathrm{mM}$ of cyclic AMP caused the re-differentiation of ovarian cancer cells into normal fibroblasts within 5 hours [8]. This morphological change was induced by the direct and rapid influence of a small amount of cyclic AMP on damaged mitochondria or microtubule (Figure 19). Notably, this differentiation inducing phenomenon has no correlation with genomic abnormalities in cancer cells.

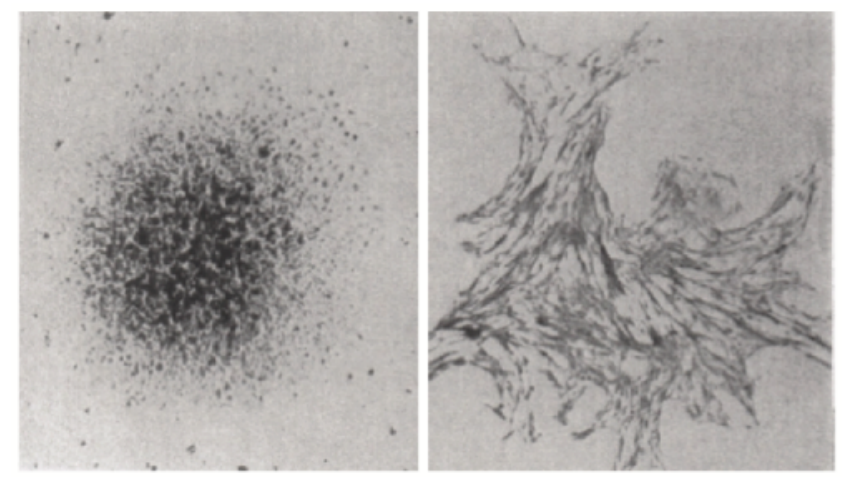

Figure 19: Ovarian cancer cells will re-differentiate to normal fibroblast by the addition of cyclic AMP cited from Hsie et al.

Oboshi et al. reported that glioma neuroblasts (NB-1 cells) could re-differentiate into normal nerve cells at 1 month after the addition of $1 \mathrm{mM}$ cyclic AMP. Thus, cyclic AMP not only has effective differentiationpromoting abilities in cancer cells, but also plays a key role in correcting defective immune-surveillance in cancer patients.

\section{Results}

In this report, the effects of SA on cancer cells and in animal and human experiments are reported. SA is a unique herbal medicine, that selectively inhibit the oxygen respiration in cancer cells, in tissue culture level and animal experiment. We could confirm for the first time that mitochondrial respiratory degeneration will be a re-differentiating factor using SA. These findings indicate that most of the hallmarks of cancer were inhibited by SA and most of them are reversible. These data demonstrate that mitochondrial respiratory degeneration is the underlying mechanism in carcinogenesis and there is no correlation with primary DNA abnormalities.
Therefore, carcinogenesis is not only due to mitochondrial respiratory degeneration but also due to a developmental degeneration as the mitochondria are deeply connected to cell differentiation. Thus, the transformation of cancer cells to immortal cells represents a change in sovereignty in the form of respiration from a higher energy production system (mitochondrial oxygen respiration) to a primitive type of bacterial fermentation system. Taken together, these findings demonstrate that cancer is a reversible disease, rather than an immortal disease caused by gene abnormalities, which can be treated by restoring the protracted mitochondria degeneration utilizing SA.

\section{Discussion}

There exist significant discrepancies between microscopic study and macroscopic study. Many oncologists have indirectly suggested that carcinogenesis is associated with degradation changes of mitochondria. However, the present research of SA showed for the first time that the inhibition of mitochondrial degeneration leads to the inhibition of carcinogenesis as described in Figure 2 of Seyfried et al.

As a report of our group carcinogenesis is dependent on the degree of degeneration according to Koch's rule. The reversibility of carcinogenesis is a important thisgs. Furthermore, Japanese and American researcher's group have reported that antioncogene are useful to investigate the evolution of multicellularity [29]. On the other hand, the degeneration of the oxygen respiration system in mitochondria should be considered an underlying cause of carcinogenesis because dedifferentiation produced reverting changes to mitochondria shape and cristae, as with cell reprogramming, Yamanaka S. et al. reported that the formation of induced pluripotent stem(iPS) cell formation involves three gene and one c-myc gene and this c-myc is oncogene which is requisite as Yamanaka factors [30]. So, cell reprogramming is one type of carcinogenesis. Fundamentally, an energy production system is a part of the cellular infrastructure. The infrastructure of the energy production system changed from a high-energy eukaryotic energy system to a lowenergy prokaryotic system which is more important for changing of cellular division system than genetic. 
Mutation from a molecular biology viewpoint, apoptosis become difficult after cytochrome $\mathrm{C}$ and calcium leaked out from mitochondria, nevertheless, according to a report by Madhavika et. al. this phenomenon is possibly a secondary event

Although it was not possible to directly prove that herbal medicine (SA) facilitated morphological change of mitochondria, but it conveyed a strong effect on lipid metabolism and which is relevant because mitochondria are mainly composed of cardiolipin which paves the inner mitochondrial membrane of cancer cell.

From an immunological standpoint, the relationship between PD-1 and PD-L1 are intimately correlated with mitochondria function. Chamoto et al. reported that there are intimate correlation with mitochondria function and PD-1 receptor [31,32]. PD-1 blockade revealed that immune activity may be intimately correlated with mitochondrial function..

As ATP is generated only in the mitochondria, degeneration of mitochondria in carcinogenesis and continual lowering of the serum content of cyclic AMP is reasonable and the phenomenon of defective immune-surveillance in cancer patients is also reasonable because of lowering concentration of cyclic AMP.

Cyclic AMP is the same molecule as ATP, but with a different energy level (12Kcal) v.s. (10 Kcal), respectively according to Professor Hayaishi Osamu who first described oxygenase. This may be the reason why a small amount of $1 \mathrm{mM}$ cyclic AMP provokes strong re-differentiation activity of cancer cells. Notably, this phenomenon has not been correlated with genetic change. Recently, one of the ingredients of herbal medicine (SA) was found to include much of the cyclic AMP. But it is not yet possible to reveal the molecular basis of herbal medicine (SA). Practitioners of western medicine are hesitant to claim that the activities of compounds are due to single substances, but, as such phenomena usually involve complex system. For example DNA replication is require four bases substances, i.e., cytidine, thymidine, adenine and guanine. Likewise, although the molecular basis is unknown, the effect of herbal medicine (SA) is important for experimental reproducibility. Thus, from a scientific standpoint, the effects of herbal medicine (SA) should not be ignored even though to molecular basis is unknown.

\section{Conclusion}

The results of this study confirmed that carcinogenesis is due to mitochondrial respiratory degeneration utilizing specific herbal medicine (SA) and carcinogenesis is reversible.

\section{Copyrights}

(c) Tsuneo Kobayashi, 2019; licensee OA Journal of Oncology. This is an Open Access article distributed under the terms of the Creative Commons Attribution License (http://creativecommons.org/ licenses/by/4.0), which permits unrestricted use, distribution, and reproduction in any medium, provided the original work is properly credited.

\section{References}

1. Warburg O. (1956) On the origin of cancer cells. Science 123:309-314.

2. Kresge N, Hanson RW, Simoni RD, et al. (2005) Sidney Weinhouse and the mechanism of ketone body synthesis from fatty acids. J Biol Chem 280:e20.

3. Burg D, Schade AL (1956) On respiratory impairment in cancer cells. Science. 124: 270-272.

4. Aisenberg AC. (1961) The glycolysis and respiration of tumors. New York Academic Press, USA.

5. Baker SG, Kramer BS (2007) Paradoxes in carcinogenesis. New opportunities for research directions. BMC Cancer. 7: 151.

6. Seyfried TN, Shelton LM. (2010) Cancer as a metabolic disease. Nutr Metab (Lond). 7:7.

7. Kobayashi T, Kawakubo T. (1994) Prospective investigation of tumor markers and risk assessment in early cancer screening. Cancer. 73:1946-1953.

8. Hsie AW, Puck TT (1971) Morphological Transformation of Chinese Hamster cells by dibutyryl adenosine cyclic 3':5'monophosphate and testosterone. Proc Natl Acad Sci U S A. 68:358-361.

9. Koura M, Isaka H, Yoshida MC, et al. (1982) Suppression of tumorigenicity in interspecific reconstituted cell and cybrids. Gann. 73:574-580.

10. Israel BA, Schaeffer WI (1987) Cytoplasmic suppression of malignancy. In vitro Cell and Dev Biol. 23:627-632.

11. Israel BA, Schaeffer WI (1988) Cytoplasmic mediation of malignancy. In vitro Cell and Dev. Biol. 24: 487-490.

12. Ristow M (2006) Oxidative metabolism in cancer growth. Curr Opin. Clin Nutri and Metab Care. 9:339-345.

13. Cuezva JM, Ortega AD, Willers I. (2009) The tumor suppressor function of mitochondria: translation into the clinics. Biochim Biophys Acta. 1792: 1145-1158.

14. Rous P (1959) Surmise and fact on the nature of cancer. Nature. 183:1357-1361.

15. Roy M, Reddy PH, lijima M (2015) Mitochondrial division and fusion in metabolism. Curr. Opin Cell Biol. 33:111-118.

16. Gray MW, Lang BF, Cedergren R, et al. (1981) Genome structure and gene content in protect mitochondrial DNAs. Nucleic Acids Res. 26: 865-879.

17. Gray MW, Burger G, Lang BF (1999) Mitochondrial evolution Science 283:1476-1481.

18. Serasinghe MN, Wieder SY, Renault TT, et al. (2015) Mitochondrial Division is Requisite to RAS-induced Transformation and Targeted by Oncogenic MAPK Pathway Inhibitors. Mol Cell. 57:521-536. 
19. Seyfried TN, Flores RE, Poff AM, et al. (2014) Cancer as a mitochondrial metabolic disease: implications for novel therapeutics. Carcinogenesis. 35:515-527.

20. Kobayashi T, Tanimizu T, et al. 1979. 32 Proceeding of J. Cell Biology, 87,( in Japanese).

21. Sugimoto K, Jo T, Tanimizu T, et al. (1982) The effect of the antitumor herb medicine" Sun Advance" in mice. Proc. Symposium. WAKAN-YAKU 15,224-227.

22. Tanimizu T, Sugimoto K, Hayashi N, et al. (1982) New approach to Chinese herb medicine, inhibition by Chinese herb medicine" Sun Advance" of SV40 transformation in mouse cells. Proc. Symposium. WAKAN-YAKU, 15, 228-233x.

23. Kobayashi T. (2018) A Blood tumor marker combination assay produces high sensitivity and high specificity for cancer according to the natural history. Cancer Med. 7:549-556.

24. Reitzer LJ, Wice BM, Kenel D. (1979) Evidence that glutamine, not sugar, is major energy source for cultured Hela cells. J Biol Chem. 254: 2669-2676.

25. Hsie AW, Puck TT. (1971) Morphological Transformation of Chinese Hamster cells by dibutyryl adenosine cyclic $3^{\prime}: 5^{\prime}$ monophosphate and testosterone. Proc Natl Acad Sci U S A. 68:358-361.

26. Sato T, Friend C, De Harven E. (1971) Ultrastructual changes in Friend erythroleucemia cells treated with Dimethyl Sulfoxide. Cancer Res. 31:1402-1417.
27. Abe H, Arichi S, Hayashi T, et al. (1979) Ultrastrucutral studies of Morris hepatoma cell reversely transformed by ginsenoside. Experimentia. 35:1647-1649.

28. Pedersen PL. (1978) Tumor mitochondria and the Bioenergetics of Cancer Cells. Prog Exp Tumor Res. 22:190-274.

29. Hanschen ER, Marriage TN, Ferris PJ, et al. (2016) The gonium pectoral genome demonstrates co-option of cell cycle regulation during the evolution of multicellularity. Nat commun. 7:11370.

30. Takahashi K, Yamada S. (2006) Induction of pluripotent stem cells from mouse embryonic and adult fibroblast cultures by defined factors. Cell. 126:663-676.

31. Kiebish MA, Han X, Cheng $H$, et al. (2008) Cardiolipin and electron transport chain abnormalities in mouse brain tumor mitochondria: lipidomic evidence supporting the Warburg theory of cancer. J lipid Res. 49:2545-2556.

32. Chamoto K, Partha S, Chowdhury A, et al. (2015) Mitochondrial activation chemicals synergize with surface receptor PD-1 blockade for $\mathrm{T}$ cell dependent anti-tumor activity. Proc Natl Acad USA. 114:E761-E770. 